\title{
Electromagnetic resonances of a multilayer metal-dielectric stack
}

\author{
M. R. Gadsdon,* J. Parsons, and J. R. Sambles \\ School of Physics, University of Exeter, Exeter, Devon, EX4 4QL, United Kingdom \\ *Corresponding author: m.r.gadsdon@exeter.ac.uk
}

Received November 21, 2008; accepted January 5, 2009;

posted January 22, 2009 (Doc. ID 104376); published March 18, 2009

\begin{abstract}
The electromagnetic resonances of multilayer metal-dielectric stacks are investigated. These structures support periodic bandpass regions, whose band edges may be predicted by considering the character of the fields inside the different layers. It is shown that the response of the structure is largely independent of its overall length, and that only the geometry of the unit cell is important. In the metal layers, the fields may have either a cosh or a sinh distribution function and match to standing waves inside the adjacent dielectric cavities at the metal-dielectric interface. It is shown that the different boundary conditions, imposed by the evanescent fields, result in the dielectric layers having a different effective length for the two modes. The sinh fields result in an effective length being very close to that of the physical length, and adjacent cavities oscillating out of phase, while the cosh fields may result in a significantly larger effective dielectric length and adjacent cavities oscillating in phase. A bandpass region is opened, with its high frequency edge always being near the dielectric Fabry-Perot limit, while the low frequency band edge is significantly redshifted. (C) 2009 Optical Society of America

OCIS codes: $230.4170,230.7408$.
\end{abstract}

\section{INTRODUCTION}

The propagation of electromagnetic radiation through metal films containing subwavelength apertures has been the subject of considerable research in the years since the discovery of surface plasmon-enhanced transmission by Ebbesen et al. [1] and Ghaemi et al. [2]. Concurrently, there has been a body of work into multilayered metaldielectric stacks, where the transmission coefficient through such stacks may be several orders of magnitude larger than that through a single layer of the metal film $[3,4]$.

The spectral response of such a structure comprises a series of photonic bandgaps (PBGs), where the reflectivity is high, separated by a series of bandpass regions where the transmissivity may be high. It has been demonstrated that near either band edge there is a large enhancement of the field intensity due to localization effects [5]. Near the high frequency band edge of the lowest frequency bandpass region, the electromagnetic field is predominantly confined to the dielectric in a manner similar to a Fabry-Perot cavity, while at the low frequency band edge it is suggested that the fields are more concentrated in the metal regions $[6,7]$.

Such metal-dielectric stacks are being investigated, both experimentally and theoretically, for a wide range of potential applications including their demonstration of negative refractive index behavior [8] and the possibility of imaging techniques associated with this property $[9,10]$, antennas embedded in windshields, electrodes on flat panel displays, electromagnetic shielding [3], and nonlinear applications [6].

While there has been significant theoretical investigation into these structures much of this work has focused on the density of states in the bandpass $[5,11]$ regions and the behavior within these regions. In this work we present a theoretical analysis of the nature of the resonant fields at each bandpass edge, and use this knowledge to demonstrate a simple yet accurate way of locating the bandpass edges of such multilayer structures. Our analysis also allows a simple physical interpretation of the behavior of metal-dielectric multilayer structures.

It should be noted that these structures also support surface plasmon polaritons when illuminated in an attenuated total reflection configuration. While this can lead to other interesting optical effects $[12,13]$, it is important to stress that all of the work presented here is at normal incidence and therefore no surface plasmon effects are observed.

\section{INITIAL RESULTS}

In Fig. 1 we present a typical reflectivity response of a multilayer structure consisting of five $6.5 \mathrm{~nm}$ thick silver layers separated by four $166 \mathrm{~nm}$ air layers. The incident and transmission materials are also air and the structure is illuminated at normal incidence. The permittivity of the silver layers are approximated by a Drude model with $\omega_{p}=1.32 \times 10^{16} \mathrm{~s}^{-1}$ and $\tau_{D}=1.45 \times 10^{14} \mathrm{~s}$. All reflectivity responses are modeled using a multilayer Fresnel calculation.

Figure 1 shows a periodic series of bandpass regions, separated by a background region (bandgap region) of high reflection. As discussed in the Introduction, there has been significant work analyzing the behavior of the stack within the bandpass region. However, the goals of the present work are to develop a better understanding of how the bandpass region itself is formed and, therefore, how its location can be predicted. To achieve these goals it 


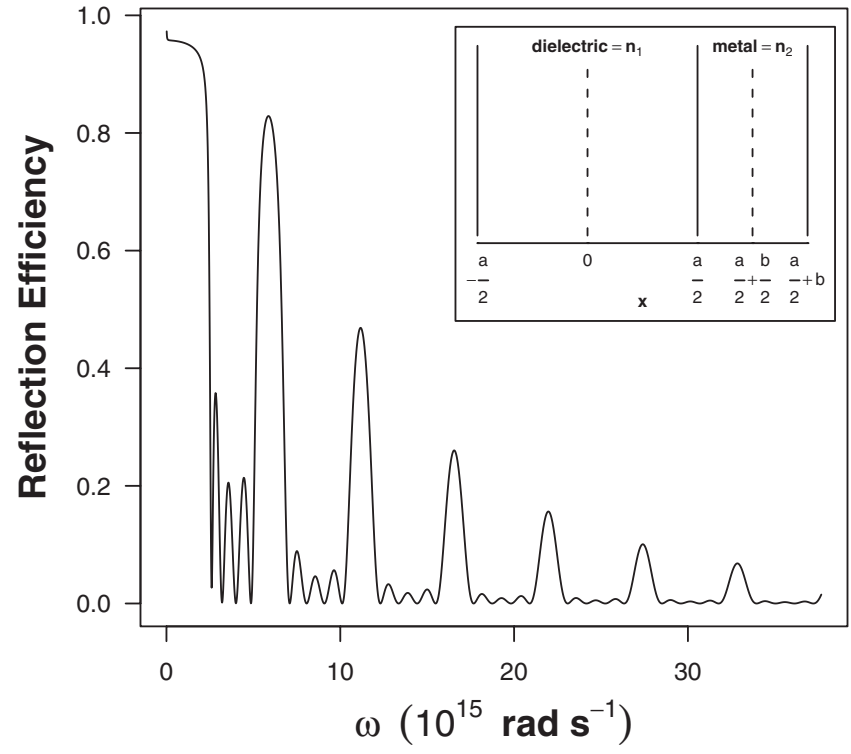

Fig. 1. Reflection efficiency response of the multilayer structure comprising five $6.5 \mathrm{~nm}$ silver layers separated by four $166 \mathrm{~nm}$ air layers. The incident and transmission materials are also air and the structure is illuminated at normal incidence. The permittivity of the silver layers are approximated by the Drude model with the parameters as defined in the main text. The frequency range is $0 \times 10^{15} \mathrm{rad} \mathrm{s}^{-1}<\omega \leqslant 37.7 \times 10^{15} \mathrm{rad} \mathrm{s}^{-1}$. The inset is a schematic representation of one unit cell of such a multilayer stack.

is important to first look at how the bandpass region disperses with a selection of parameters.

The obvious parameter to change first is the number of layers of metal (silver in this case). The reflectivity response of two structures, one with ten layers of silver, the

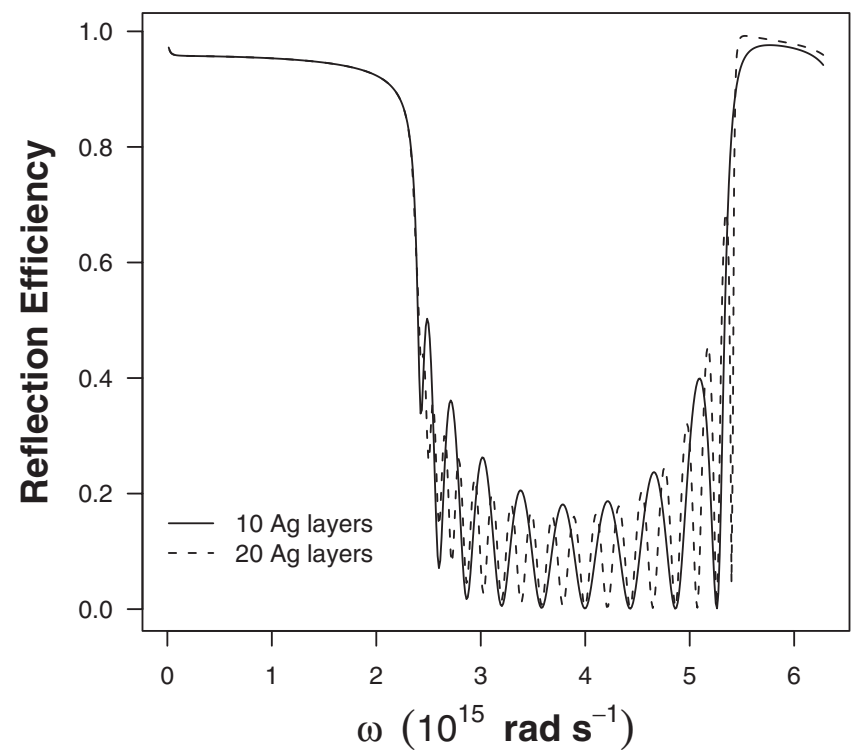

Fig. 2. Reflection efficiency response of the multilayer structure comprising ten $6.5 \mathrm{~nm}$ silver layers separated by nine $166 \mathrm{~nm}$ air layers (solid curve) and twenty $6.5 \mathrm{~nm}$ silver layers separated by nineteen $166 \mathrm{~nm}$ air layers (dashed curve). The incident and transmission materials are also air and the structure is illuminated at normal incidence. The permittivity of the silver layers are approximated by the Drude model with the parameters as defined in the main text. The frequency range is $0 \times 10^{15} \mathrm{rad} \mathrm{s}^{-1}$ $<\omega \leqslant 6.28 \times 10^{15} \mathrm{rad} \mathrm{s}^{-1}$. other with 20 layers, are presented in Fig. 2; we show only the lowest frequency bandpass region for clarity.

It is evident from Fig. 2 that doubling the number of metal layers in the structure sharpens the edges of the bandpass region. The extra layers of metal also slightly increases the overall absorption of the structure and, of course, introduces correspondingly more subresonances in the bandpass region. However, the bandwidth of the resonance is independent of the overall length of the structure and, therefore, depends only on the unit cell of each layer.

It has been shown previously that resonant tunneling associated with strong transmission may give strong fields inside the metal rather than the dielectric [6]. Other work has shown how this resonant tunneling may give propagating fields through the metal dielectric stack [7]. To establish that the imaginary part of the metal permittivity has no important role, we present in Fig. 3 a comparison between a stack comprising silver, with its permittivity approximated by the Drude model as before, and that with the imaginary part of its permittivity removed. Removing the imaginary part of the permittivity from the calculation, leaving only a negative real part, makes the fields inside the metal purely evanescent because the refractive index becomes purely imaginary.

In Fig. 3 it is clear, apart from the obvious effects of removing the absorption of the structure, that removing the imaginary part of the permittivity and hence making the fields inside the metal purely evanescent causes no other effect on the bandpass resonance and its spectral position. Therefore, it is the evanescent character of the fields inside the metal layers that plays the significant role in resonant tunneling. The conclusions that the bandpass

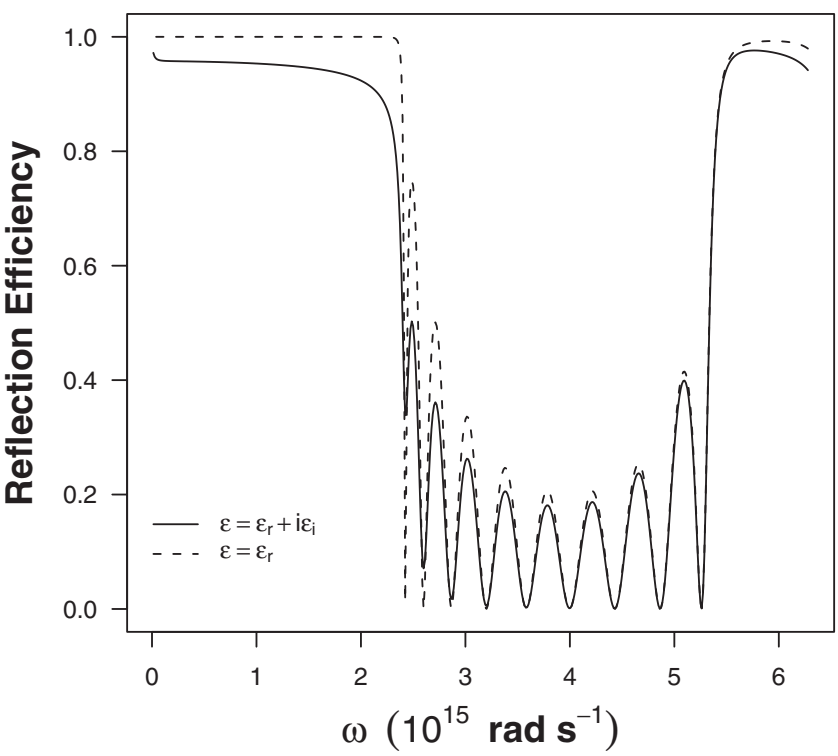

Fig. 3. Reflection efficiency response of the multilayer structure comprising ten $6.5 \mathrm{~nm}$ silver layers separated by nine $166 \mathrm{~nm}$ air layers. The incident and transmission materials are also air and the structure is illuminated at normal incidence. The permittivity of the silver layers are approximated by the Drude model with the parameters as defined in the main text (solid curve) and by the Drude model with the imaginary part of the permittivity removed (dashed curve). The frequency range is $0 \times 10^{15} \mathrm{rad} \mathrm{s}^{-1}$ $<\omega \leqslant 6.28 \times 10^{15} \mathrm{rad} \mathrm{s}^{-1}$. 
regions depend only on the unit cell of the structure, and that only the evanescent fields within the metal are important, allows a simple mathematical analysis to be formulated.

\section{THEORETICAL ANALYSIS}

A generic multilayer structure may be divided into a set of unit cells, as shown in the inset of Fig. 1, which is a schematic representation of one unit cell of a multilayer stack comprising two different materials of thickness $a$ and $b$. The refractive index of the dielectric of thickness $a$ is $n_{1}$, and the refractive index of the metal of thickness $b$ is given by $n_{2}=\sqrt{\epsilon_{r}}=i k_{2}$. We have omitted the imaginary part of the permittivity so that the fields inside the metal are purely evanescent, as justified by the result in Fig. 3.

The possible resonances of the structure occur when standing waves exist in the dielectric layers; these standing waves must then be matched with the evanescent fields inside the metal layers. The amplitude of the tangential $\underline{E}$ field and its derivative with respect to $x$ must be continuous across each boundary. From symmetry arguments, a cos or a sin wave function is expected, as the limit solutions, about either $x=0$ and/or $x=a / 2+b / 2$, respectively. Therefore, there are four expressions that describe the possible wave functions that may specify the band edges:

$$
\begin{aligned}
& \psi_{n_{1}}=A \cos \left(n_{1} k_{0} x\right), \\
& \psi_{n_{1}}=A \sin \left(n_{1} k_{0} x\right), \\
& \psi_{k_{2}}=B \cosh \left(k_{2} k_{0}\left[x-\left(\frac{a}{2}+\frac{b}{2}\right)\right]\right), \\
& \psi_{k_{2}}=-B \sinh \left(k_{2} k_{0}\left[x-\left(\frac{a}{2}+\frac{b}{2}\right)\right]\right),
\end{aligned}
$$

where $\psi_{k_{1}}$ and $\psi_{k_{2}}$ represent the wave functions, and $A$ and $B$ are the amplitude coefficients of the wave functions, in adjacent layers of the stack with refractive indices $n_{1}$ and $k_{2}$, respectively. $k_{0}=\omega / c$ is the wave vector, $\omega$ is the angular frequency, and $c$ is the speed of light in the incidence medium, assumed to be vacuum. The derivatives of Eqs. (1)-(4) with respect to $x$ are thus

$$
\begin{aligned}
& \frac{\partial \psi_{n_{1}}}{\partial x}=-n_{1} k_{0} A \sin \left(n_{1} k_{0} x\right), \\
& \frac{\partial \psi_{n_{1}}}{\partial x}=n_{1} k_{0} A \cos \left(n_{1} k_{0} x\right), \\
& \frac{\partial \psi_{k_{2}}}{\partial x}=k_{2} k_{0} B \sinh \left(k_{2} k_{0}\left[x-\left(\frac{a}{2}+\frac{b}{2}\right)\right]\right), \\
& \frac{\partial \psi_{k_{2}}}{\partial x}=-k_{2} k_{0} B \cosh \left(k_{2} k_{0}\left[x-\left(\frac{a}{2}+\frac{b}{2}\right)\right]\right) .
\end{aligned}
$$

Supposing the wave functions in the media are of a cos and cosh form, then Eqs. (1), (3), (5), and (7), must be equated:

$$
\begin{gathered}
A \cos \left(n_{1} k_{0} \frac{a}{2}\right)=B \cosh \left(k_{2} k_{0}\left[-\frac{b}{2}\right]\right), \\
-n_{1} k_{0} A \sin \left(n_{1} k_{0} \frac{a}{2}\right)=k_{2} k_{0} B \sinh \left(k_{2} k_{0}\left[-\frac{b}{2}\right]\right) .
\end{gathered}
$$

Dividing Eq. (10) by (9) eliminates the unknown coefficients $A$ and $B$, giving a single equation:

$$
n_{1} \tan \left(n_{1} k_{0} \frac{a}{2}\right)=k_{2} \tanh \left(k_{2} k_{0} \frac{b}{2}\right) .
$$

This process may be repeated for all the possible wave functions in the adjacent layers, yielding three more equations:

$$
\begin{aligned}
& n_{1} \tan \left(n_{1} k_{0} \frac{a}{2}\right)=k_{2} \operatorname{coth}\left(k_{2} k_{0} \frac{b}{2}\right), \\
& n_{1} \cot \left(n_{1} k_{0} \frac{a}{2}\right)=-k_{2} \tanh \left(k_{2} k_{0} \frac{b}{2}\right), \\
& n_{1} \cot \left(n_{1} k_{0} \frac{a}{2}\right)=-k_{2} \operatorname{coth}\left(k_{2} k_{0} \frac{b}{2}\right) .
\end{aligned}
$$

The solutions to these equations [(11)-(14)] then define the location of the band edge resonances of the infinite multilayer structure.

\section{FURTHER RESULTS}

Figure 4 is a plot of the $\underline{E}$ fields for a finite structure with the same parameters as in Fig. 1, except the metal thickness is $b=20 \mathrm{~nm}$ and the air thickness is $a=150 \mathrm{~nm}$ for clarity. The fields are plotted at two frequencies, the lowest and highest frequency reflection minima, and the dashed boxes outline the location of the metal layers. The field plots confirm the localization of the fields in the dielectric and the strong fields within the metal for the low frequency band edge. This is evidence of an important feature of the bandpass region at either edge: at the high frequency band edge, the field in each dielectric cavity oscillates out of phase with its adjacent cavities whereas, at the low frequency band edge, the fields in adjacent cavities oscillate in phase.

By comparing Fig. 4 with Eq. (11)-(14) the nature of the distribution function of the fields in both the metal and the dielectric layers may be determined. At the high frequency band edge, where adjacent cavities oscillate out of phase, there is only a small (but not negligible) amount of the fields of each oscillation within the metal. The high frequency edge is described by Eq. (12), which originates from a cos oscillation in the dielectric layers and a sinh distribution function in the metal layers; it corresponds to a sequential series of coupled Fabry-Perot cavities. However, it occurs at a slightly lower frequency than the 


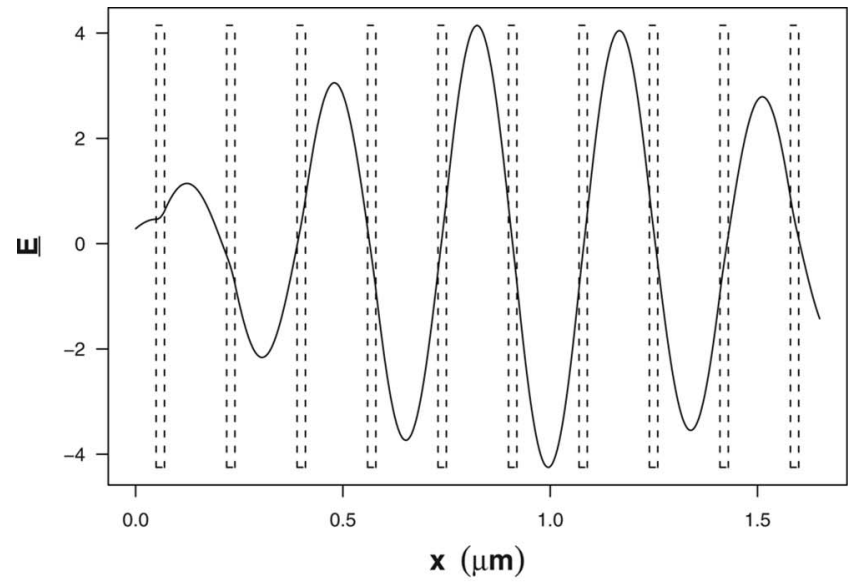

(a) $\omega=5.58 \times 10^{15} \mathrm{rad} \mathrm{s}^{-1}$

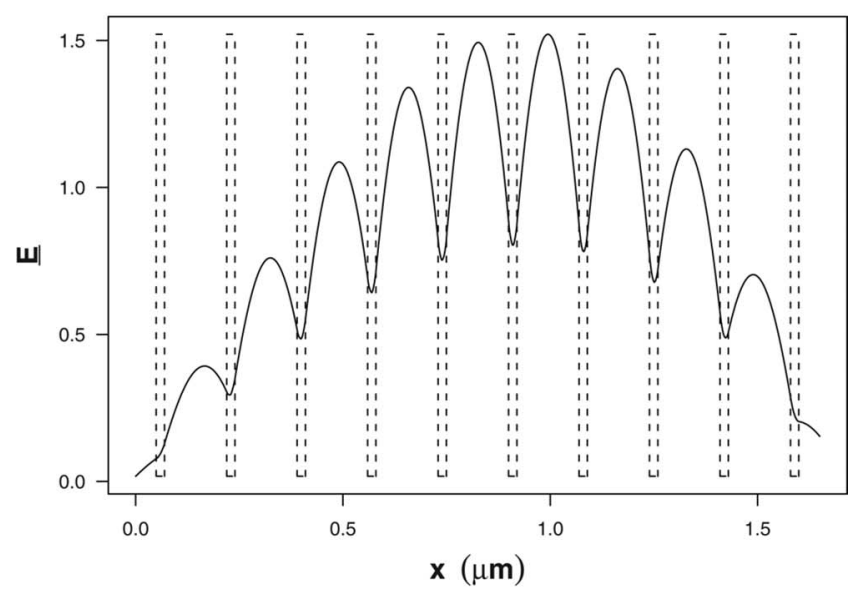

(b) $\quad \omega=3.73 \times 10^{15} \mathrm{rad} \mathrm{s}^{-1}$

Fig. 4. $\quad$ E fields for a multilayer structure, comprising ten $20 \mathrm{~nm}$ silver layers separated by nine $150 \mathrm{~nm}$ air layers. The incident and transmission materials are also air and the structure is illuminated at normal incidence and the permittivity of the silver layers are approximated by the Drude model with parameters as defined in the main text (including the imaginary part). The fields are plotted at two frequencies corresponding to the highest and lowest frequency reflection minima of the first bandpass region. The dashed lines indicate the location of the metal layers.

simple dielectric thickness Fabry-Perot limit due to the finite conductivity of the metal [14].

At the low frequency band edge, where adjacent cavities oscillate in phase, a significant proportion of the fields of each oscillation is within each of the metal regions, thereby increasing the effective length of each unit cell and resulting in a redshift in the band edge. This may be described by Eq. (11), which originates from a cos oscillation in the dielectric layers and a cosh distribution function in the metal layers.

Equations (11) and (12) may be used to plot the field profiles of two adjacent unit cells for an infinite multilayer structure, to confirm the pairing of the equations with the field plots of Fig. 4 . Figure 5 is plotted by using Eq. (1) and (3) in conjunction with the solutions determined from Eqs. (11) and (12), which as expected give the same limit frequencies as in Fig. 4. The metal thickness is $b=20 \mathrm{~nm}$ and the air thickness is $a=150 \mathrm{~nm}$.
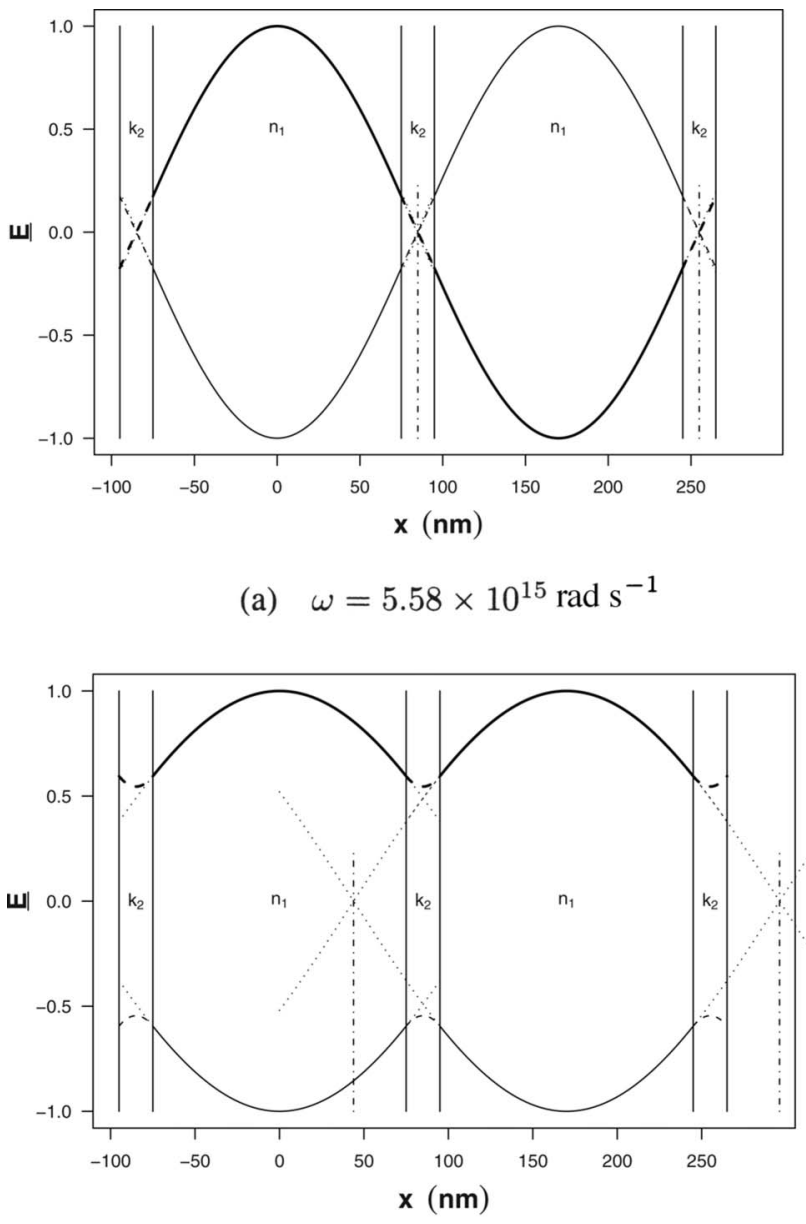

(b) $\omega=3.73 \times 10^{15} \mathrm{rad} \mathrm{s}^{-1}$

Fig. 5. $\quad$ E fields for an infinite multilayer structure, comprising $20 \mathrm{~nm}$ silver layers separated by $150 \mathrm{~nm}$ air layers. The incident and transmission materials are also air and the structure is illuminated at normal incidence. The permittivity of the silver layers are approximated by the Drude model, using the parameters defined in the main text, with the imaginary part removed. The fields are plotted (a) using Eqs. (1) and (3) and the solution to Eq. (12), and (b) using Eqs. (1) and (3) and the solution to Eq. (11), which correspond to the band edges of Fig. 4. The solid lines are the cos standing waves in the dielectric, the dashed lines are the (a) sinh and (b) cosh waves in the metal, and the dotted lines show where the dielectric standing waves would continue if no metal was present. The bold and narrow curves are $\pi$ out of phase and the vertical dotted-dashed lines indicate the effective cavity length.

Comparing Figs. 4 and 5 clearly shows that the two plots agree qualitatively. The plots relating to the low frequency band edge [Figs. 4(b) and 5(b)] both show that the fields in the cavities oscillate in phase, and that the fields inside the metal layers have a cosh profile. Both of the plots corresponding to the high frequency edge [Figs. 4(a) and 5(a)] illustrate that adjacent cavities oscillate out of phase, and that the filed profile in the metal has a sinh form. The only differences between the two plots arise from the fact that slightly different parameters are used, and that the five layer structure is not able to form exact cos, cosh, and sinh waves due to its finite number of cavities. This confirms that the equations formulated, for the description of the fields inside the metal regions as being evanescent standing fields, are valid. 
Note that in Fig. 5, and all subsequent infinite structure field plots (Figs. 7 and 8), a fixed cavity resonance amplitude coefficient, $A=1$, is implemented for simplicity so that any parameter changes are manifested in only the gradients of the cavity resonances and evanescent field amplitudes at the interface.

One further interesting observation can be made from these field plots. It was stated previously that the FabryPerot-like high frequency band edge has adjacent cavities that oscillate out of phase, while the low frequency band edge has adjacent cavities that oscillate in phase. This results in the fields inside the metal, for the low frequency band edge, never going through zero and the resonance is a dc-like resonance throughout the entire structure.

Figure 6 presents the dispersion of a similar bandpass region, as shown in Fig. 3, as a function of the real part of the permittivity of the metal layers. Naturally, the high frequency band edge, where the fields in the metal are weak and the cavities are Fabry-Perot-like, is largely independent of $\epsilon_{r}$ of the metal layers, whereas the low frequency band edge, where the fields are stronger in the metal, is highly sensitive to $\epsilon_{r}$. There is a small dependency of the high frequency edge on $\epsilon_{r}$, due to the finite conductivity; however, once $\epsilon_{r}$ is very large, the resonance blueshifts to exactly the Fabry-Perot resonance. It has already been stated that the high frequency band edge relates to the solution to Eq. (12) and the solutions to this equation are shown in Fig. 6 as the upper series of black squares. Clearly, the solution to the equation accurately predicts the spectral location of the high frequency band edge and, therefore, the description of the evanescent fields, emanating from either boundary, interacting in the metal to form a sinh distribution function is valid.

The low frequency edge relates to the solution to Eq. (11). Again, the solution (lower series of black squares) accurately predicts the spectral location of the low frequency band edge, validating the description of the fields in the metal as standing evanescent fields with a cosh dis-

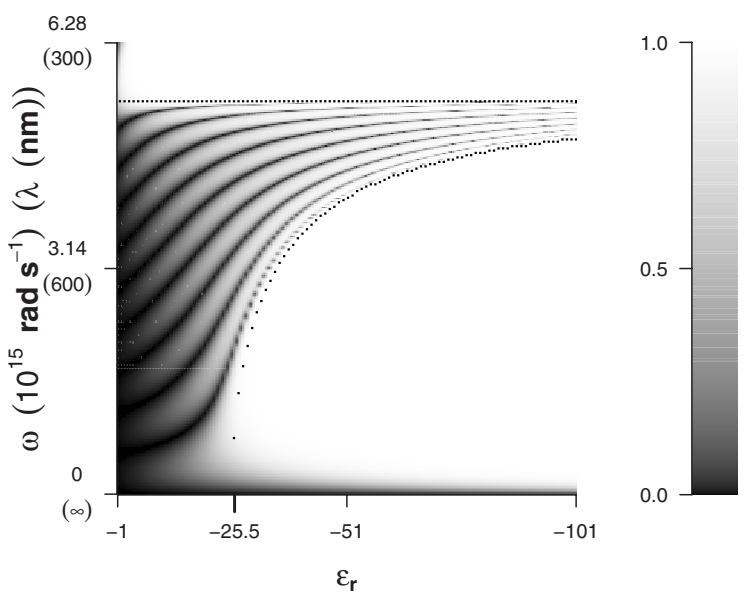

Fig. 6. Reflection efficiency response of the multilayer structure, comprising ten $6.5 \mathrm{~nm}$ silver layers separated by nine $166 \mathrm{~nm}$ air layers, as a function of the real part of the dielectric permittivity. The black squares are the limit solutions to Eqs. (11) and (12) for the same permittivities. The incident and transmission materials are also air and the structure is illuminated at normal incidence. The frequency range is $0 \times 10^{15} \mathrm{rad} \mathrm{s}^{-1}<\omega$ $\leqslant 6.28 \times 10^{15} \mathrm{rad} \mathrm{s}^{-1}(\infty>\lambda \geqslant 300 \mathrm{~nm})$, and the permittivity is defined as $-101 \leqslant \epsilon_{r} \leqslant-1$. tribution function. The slight deviation from the solution, which occurs at low $\epsilon_{r}$, is due to the modeled metaldielectric structure being finite, therefore, the standing waves in the cavities and fields in the metal layers are not able to form standing fields with exactly $\cos (h)$ or $\sin (h)$ distribution functions.

Relating Figs. 5 and 6 back to Eqs. (11) and (12), it is possible to give a rigorous interpretation of the effect of varying $\epsilon_{r}$. The high frequency band edge, given by Eq. (12), does not significantly change in frequency as $k_{2}$ is reduced from high values $\left(k_{2}^{2}=\left|\epsilon_{r}\right|\right)$. This is essentially because, for small value of $k_{2}\left[k_{2} k_{0}(b / 2) \ll 1\right]$, coth $\rightarrow \infty$ and, thus, $n_{1} k_{0}(a / 2) \simeq \pi / 2$, or $\lambda_{0}$ is, as expected, $\simeq 2 a n_{1}$. For large values of $k_{2}\left[k_{2} k_{0}(b / 2) \gg 1\right], \operatorname{coth} \rightarrow 1$, and thus, $\tan \left[n_{1} k_{0}(a / 2)\right]=k_{2} / n_{1}$. As $k_{2} \gg n_{1}$ we again arrive at $n_{1} k_{0}(a / 2) \simeq \pi / 2$, and $\lambda_{0}$ is still $\simeq 2 a n_{1}$. Therefore, for all values of $k_{2}, k_{2} \operatorname{coth}\left[k_{2} k_{0}(b / 2)\right]>1$ and thus, $n_{1} k_{0}(a / 2)$ $\simeq \pi / 2$. Any change there is in the high is in the high frequency edge will be a very small decrease with decreasing $k_{2}$ because $k_{2} \operatorname{coth}\left[k_{2} k_{0}(b / 2)\right]$ does not $\rightarrow \infty$ as quickly for small $k_{2}$ as it does for large $k_{2}$. The exact solution is, therefore, significantly dependent on $n_{1}$ and $a$ and also slightly dependent on the relative values of $k_{2}$ and $b$.

The low frequency edge, given by Eq. (11), does change markedly in frequency as $k_{2}$ is reduced. This is because, in contrast to coth, for small $k_{2}\left[k_{2} k_{0}(b / 2) \ll 1\right]$, $\tanh \left[k_{2} k_{0}(b / 2)\right] \rightarrow k_{2} k_{0}(b / 2)$, and thus, there is a cut-off $\epsilon_{r}$ value given by $n_{1}^{2} k_{0}(a / 2) \simeq k_{2}^{2} k_{0}(b / 2)$ or $\epsilon_{r} \simeq n_{1}^{2}(a / b)$ $=-25.5$, as indicated in Fig. 6. While, for large $k_{2}\left[k_{2} k_{0}(b / 2) \gg 1\right], \tanh \left[k_{2} k_{0}(b / 2)\right] \rightarrow 1$ and we arrive at the same solution for the high frequency band edge, $\lambda_{0}$ $\simeq 2 a n_{1}$. Thus, when $k_{2} k_{0}(b / 2) \gg 1$, the bandwidth of the bandpass region has been reduced significantly such that it approaches a single resonance. Clearly, the low frequency band edge is much more sensitive to changes in $k_{2} k_{0}(b / 2)$ than the high frequency band edge. Therefore, provided $k_{2} k_{0}(b / 2) \ngtr 1$, the exact solution is significantly dependent on the relative values of $k_{2}$ and $b$ as well as $n_{1}$ and $a$.

This behavior may also be thought of as the sensitivity of the effective cavity length, resulting from the cosh and sinh evanescent fields, to $\epsilon_{r}$ and is shown in Figs. 5, 7, and 8 . They show that, for small $k_{2}\left[k_{2} k_{0}(b / 2) \ll 1\right]$, the gradient of the sinh fields at the interface $\rightarrow A \sqrt{\epsilon_{r}} k_{0}$, while the gradient of the $\cosh$ fields $\rightarrow A \epsilon_{r} k_{0}^{2}(b / 2)$. Therefore, for a given $A$ in this regime, the gradient of the sinh fields at the interface are always larger and less sensitive to changes in $\epsilon_{r}$ than the cosh fields.

Furthermore, the gradient of the sinh fields, at the interface, are always similar to the largest values that the cavity resonance may obtain, regardless of $k_{2} k_{0}(b / 2)$. The gradient of the cavity resonance could be increased by increasing $A$, but this would inherently increase the amplitude coefficient of the sinh fields and so their gradient in turn. Therefore, the sinh evanescent fields may only match to the part of the cavity resonance with a relatively large gradient. This results in the majority of the cavity standing wave being excluded from the metal regions, causing the effective cavity length to be approximately equal to the physical cavity length, therefore the high frequency band edge occurs at almost exactly the FabryPerot limit. This can be seen in Fig. 5(a) and both plots in 


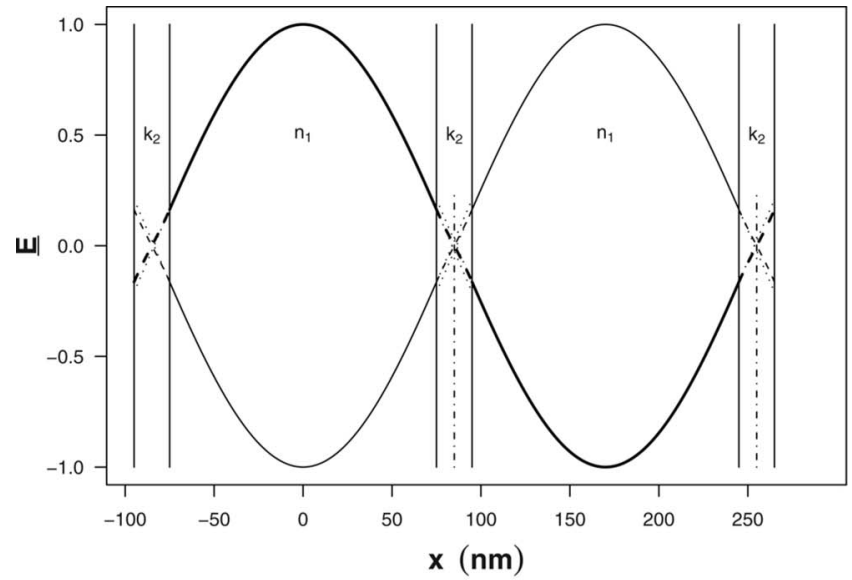

(a) $\epsilon_{r}=-40 \rightarrow \omega=5.75 \times 10^{15} \mathrm{rad} \mathrm{s}^{-1}$

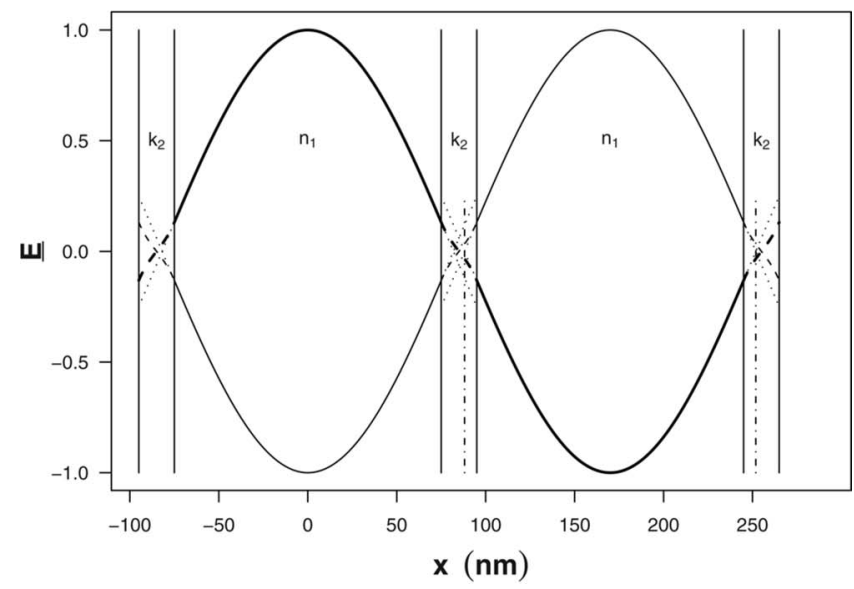

(b) $\epsilon_{r}=-11.5 \rightarrow \omega=5.62 \times 10^{15} \mathrm{rad} \mathrm{s}^{-1}$

Fig. 7. $\quad$ E fields for the high frequency band edge of an infinite multilayer structure, comprising $20 \mathrm{~nm}$ silver layers separated by $150 \mathrm{~nm}$ air layers. The incident and transmission materials are also air, the structure is illuminated at normal incidence, and the permittivity of the silver layers are fixed at either $\epsilon_{r}=(\mathrm{a})-40$ or (b) -11.5. The fields are plotted using Eqs. (1) and (3) and the solutions to Eq. (12). The solid lines are the cos standing waves in the dielectric, the dashed lines are the sinh waves in the metal, and the dotted lines show where the dielectric standing waves would continue if no metal was present. The bold and narrow curves are $\pi$ out of phase and the vertical dotted-dashed lines indicate the effective cavity length.

Fig. 7, where the dotted lines illustrate how the fields of the cavity resonances would continue were no metal present, the vertical dotted-dashed lines denoting the effective cavity length. As expected, the high frequency band edge tends to exactly the Fabry-Perot limit as $\left|\epsilon_{r}\right| \rightarrow \infty$.

In contrast, in the regime $\left[k_{2} k_{0}(b / 2) \ll 1\right]$, the gradient of the cosh evanescent standing fields, at the interface, may have a gradient similar to the lower values the cavity resonance may obtain. Again, $A$ could be reduced to reduce the gradient of the cavity resonance, but this would in turn reduce the amplitude coefficient and hence the gradient of the cosh fields. Therefore, these fields may match to a part of the adjacent standing wave with a relatively small gradient, effectively removing a significant

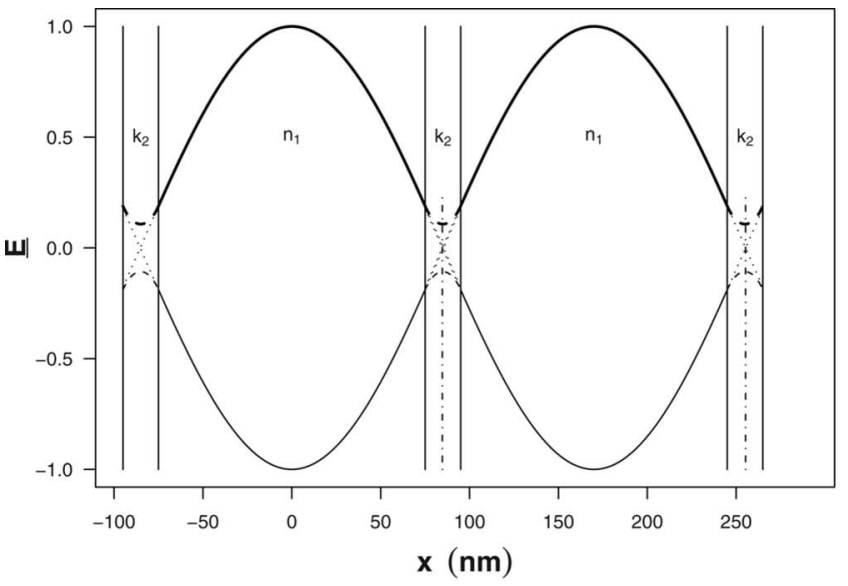

(a) $\epsilon_{r}=-40 \rightarrow \omega=5.52 \times 10^{15} \mathrm{rad} \mathrm{s}^{-1}$

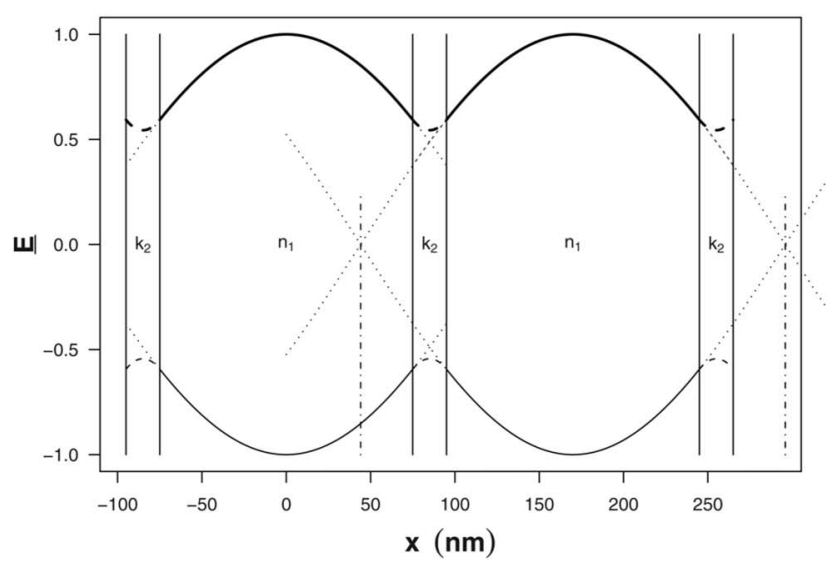

(b) $\epsilon_{r}=-11.5 \rightarrow \omega=3.74 \times 10^{15} \mathrm{rad} \mathrm{s}^{-1}$

Fig. 8. $\quad \underline{\mathbf{E}}$ fields for the low frequency band edge of an infinite multilayer structure, comprising $20 \mathrm{~nm}$ silver layers separated by $150 \mathrm{~nm}$ air layers. The incident and transmission materials are also air and the structure is illuminated at normal incidence and the permittivity of the silver layers are fixed at either $\epsilon_{r}$ $=$ (a) -40 or (b) -11.5 . The fields are plotted using Eqs. (1) and (3) and the solutions to Eqs. (11). The solid lines are the cos standing waves in the dielectric, the dashed lines are the cosh waves in the metal, and the dotted lines show where the dielectric standing waves would continue if no metal was present. The bold and narrow curves are $\pi$ out of phase and the vertical dotted-dashed lines indicate the effective cavity length.

proportion of the cavity standing waves. This results in the effective cavity length being significantly larger than the physical cavity length, shown in Figs. 5(b) and 8(b), and a redshift in the low frequency band edge occurs.

Finally, for large $k_{2}\left[k_{2} k_{0}(b / 2) \gg 1\right]$, the gradients of the sinh and cosh fields at the interface $\rightarrow \infty$ at the same rate. Therefore the gradients of both these fields are similar to the largest values the cavity resonance may obtain, and the effective cavity length is always approximately the dielectric thickness and largely independent of $k_{2}$ for both field distributions, as is shown in Figs. 7(a) and 8(a). Therefore, the bandpass region narrows as the two solutions converge.

Thus, it is the different boundary conditions imposed at the interface between the metal and dielectric layers by 
the evanescent fields inside the metal, with either a cosh or a sinh distribution function, which determines the effective cavity length and thus the resonant frequency.

Inside the bandpass region the fields are more complicated. However, from Eqs. (11) and (12), it is straightforward to see that moving from the low frequency band edge, through the bandpass region, to the high frequency band edge results in the evanescent standing fields inside the metal layers morphing from a cosh to a sinh distribution function, and adjacent cavities going from in phase to out of phase oscillations.

The limit solutions for the case when $k_{2} k_{0}(b / 2) \gg 1$ demonstrates some important conclusions. In this regime both band edges are almost exactly at the dielectric Fabry-Perot limit. Therefore, the bandpass region approaches a single resonance and is completely independent of both the thickness of the metal layers and their permittivites. Furthermore, the results of Fig. 2 show the interesting fact that the bandpass regions of the structures, at all frequencies, only depend on the unit cell and are independent of the number of cavities, and therefore the total extent of the structure. The only difference introduced by including more cavities is the effect of extra absorption, and a sharpening of the bandpass regions as the structure tends to the idealized infinite structure.

We now extend the frequency range of the investigation in Fig. 6 to observe the solutions to the other equations, (13) and (14). Figure 9 shows the several bandpass regions observed in Fig. 1. The two lowest bandpass regions are the regions bounded by the solutions to Eqs. (11) and (12), and (13) and (14), the higher frequency bandpass regions are then the higher orders of each of these bounded regions. The mathematical analysis can be repeated for the behavior of each bandpass region to obtain information on the distribution function of the standing fields in the layers. The first bandpass region was explained above, but, for example, the second bandpass region

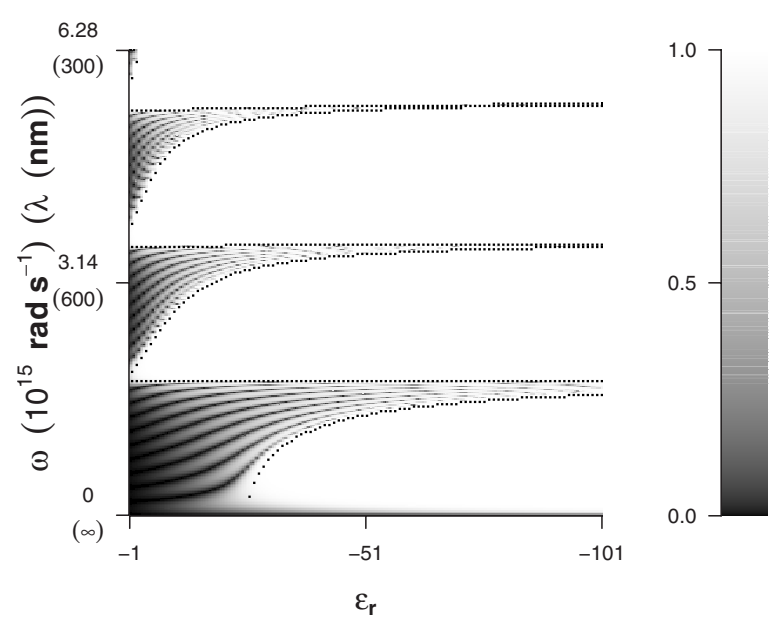

Fig. 9. Reflection efficiency response of the multilayer structure, comprising ten $6.5 \mathrm{~nm}$ silver layers separated by nine $166 \mathrm{~nm}$ air layers, as a function of the real part of the dielectric permittivity. The black squares are the limit solutions to Eqs. (11)-(14) (and their harmonic solutions) for the same permittivities. The incident and transmission materials are also air and the structure is illuminated at normal incidence. The frequency range is $0 \times 10^{15} \mathrm{rad} \mathrm{s}^{-1}<\omega \leqslant 18.84 \times 10^{15} \mathrm{rad} \mathrm{s}^{-1} \quad(\infty>\lambda$ $\geqslant 100 \mathrm{~nm}$ ), and the permittivity is defined as $-101 \leqslant \epsilon_{r} \leqslant-1$. yields a similar discussion, except in this case the standing wave in the dielectric has a sin rather than a cos wave function. It should be noted that the higher order solutions are only higher harmonics of the cos and sin standing waves in the cavities, because the evanescent waves in the metal, which interact to form cosh and sinh distribution functions, cannot have higher orders.

\section{GENERALIZATION}

It is straightforward to generalize the theoretical analysis to real materials. Equations (11)-(14) must be modified such that the metal layers may have a complex permittivity, $k_{2} \rightarrow n_{2}=n+i k$, the resulting equations are

$$
\begin{aligned}
& n_{1} \tan \left(n_{1} k_{0} \frac{a}{2}\right)=-n_{2} \tan \left(n_{2} k_{0} \frac{b}{2}\right), \\
& n_{1} \tan \left(n_{1} k_{0} \frac{a}{2}\right)=n_{2} \cot \left(n_{2} k_{0} \frac{b}{2}\right), \\
& n_{1} \cot \left(n_{1} k_{0} \frac{a}{2}\right)=n_{2} \tan \left(n_{2} k_{0} \frac{b}{2}\right), \\
& n_{1} \cot \left(n_{1} k_{0} \frac{a}{2}\right)=-n_{2} \cot \left(n_{2} k_{0} \frac{b}{2}\right) .
\end{aligned}
$$

However, the solutions $\omega$ will be complex and the absolute magnitude must be taken. As an example, Fig. 10 presents the results of varying the thickness of the metal in a real silver-dielectric stack, the thickness range being $1 \mathrm{~nm} \leqslant b \leqslant 50 \mathrm{~nm}$.

From Fig. 10 it is clear that the solutions to the generalized equations (black squares) accurately predict the locations of the band edges for real metals with complex

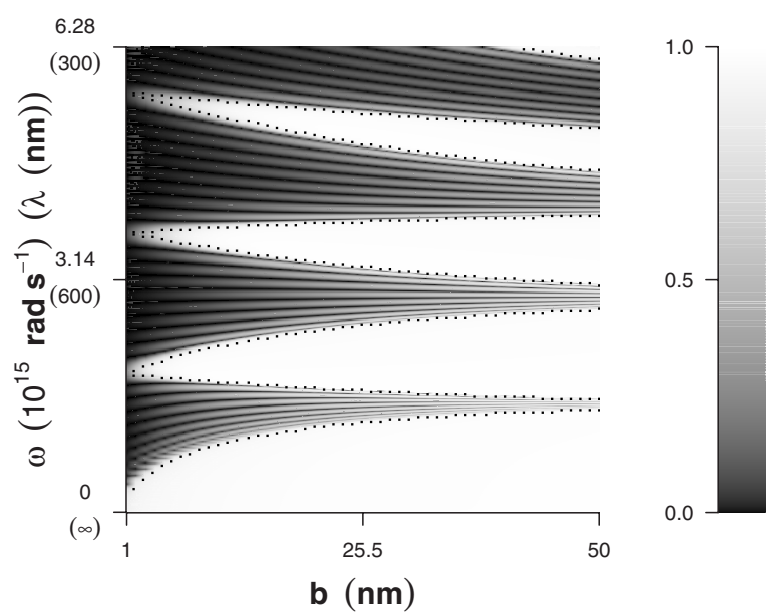

Fig. 10. Reflection efficiency response of the multilayer structure, comprising ten silver layers of thickness $1 \mathrm{~nm} \leqslant b \leqslant 50 \mathrm{~nm}$ separated by nine $166 \mathrm{~nm}$ air layers, as a function of the thickness of the silver layers. The black squares are the limit solutions to Eqs. (15)-(18) (and their harmonic solutions). The incident and transmission materials are also air and the structure is illuminated at normal incidence. The frequency range is $0 \times 10^{15} \mathrm{rad} \mathrm{s}^{-1}<\omega \leqslant 18.84 \times 10^{15} \mathrm{rad} \mathrm{s}^{-1} \quad(\infty>\lambda \geqslant 100 \mathrm{~nm})$, and the permittivity is defined by the Drude model with parameters as defined in the main text. 
permitivities. Similar boundary condition arguments as those discussed for Fig. 6 explain the dispersion of the modes for varying metal thickness. For example, the solution to Eq. (11) (the bottom solution), can be understood because increasing the width of the metal layers $b$ has a similar effect to increasing $k_{2}=\sqrt{\epsilon_{r}}$.

\section{CONCLUSIONS}

The reflectivity response of metal-dielectric stacks has been investigated. It has been demonstrated that these structures may exhibit a wide bandpass region in their response, and that the spectral location of this region is independent of the number of layers in the structure, implying that only the unit cell need be considered in a theoretical analysis. The results of previous work, such as the localization of the fields at either edge of the observed bandpass regions, has been confirmed.

A series of equations have been formulated that describe the possible field distribution functions that may exist inside the metal and dielectric layers. These equations have been idealized for metals with only real permittivities so that the fields inside the metal are then purely evanescent. The solutions to these equations have been shown to accurately predict all of the possible bandpass regions that the structure may support. In doing so it has been shown that the resonant tunneling that is allowed in the bandpass regions is due only to the evanescent character of the fields in the metal layers coupling to cavity resonances in the dielectric. The evanescent fields, which emanate from either boundary of the metal layers, interact to form evanescent standing fields with either a cosh or a sinh distribution function. For the limit solutions, the evanescent standing fields at the boundaries must match to the standing cos or sin waves oscillating in the adjacent cavities. For a given cavity resonance amplitude coefficient, the gradient of the sinh or cosh evanescent standing fields may have either a relatively large or a small gradient at the boundary. Therefore, they may match to either a large gradient or a lower gradient part of the adjacent oscillation, respectively. This results in the sinh field exluding the majority of the cavity oscillation from the metal, and adjacent cavities oscillating out of phase, so that the effective cavity length is very close to the physical cavity length and the structure behaves as a series of Fabry-Perot cavities. The cosh field removes a significant proportion of the cavity oscillation, with adjacent cavities oscillating in phase. This allows a longer wavelength oscillation to exist inside the cavity, increasing the effective cavity length and leading to a redshift in the resonance away from the Fabry-Perot limit. It is the boundary conditions imposed by the evanescent fields that determine the effective cavity length of the structure, and hence the frequency of the resonances. Furthermore, the cosh evanescent fields are significantly more sensitive to changes in the parameters of the structure than the sinh fields.

It has also been shown that, at all frequencies, the bandpass regions of the structures are independent of the total number of cavities, therefore the overall extent of the structures, and only depend upon the geometry of the unit cell. An expression for the high frequency limit of the low frequency band edge has been obtained analytically, provided the limit is in the regime where $k_{2} \gg n_{1}$. Combining this with the independence of the total number of cavities demonstrates that the response of thin metal layer-dielectric multilayer structures in this regime are independent of the metal thickness (provided $b \ll \lambda_{0}$ ), permittivity, and number of layers.

The analysis presented here applies to any system where evanescent waves must match to propagating waves - for example, nondiffracting hole arrays below the hole cut off frequency. This presents the interesting idea where, by perforating a metal film with subwavelength and nonresonating holes (or indeed adding subwavelength nonresonating particles to a dielectric layer), such that the film can be treated by effective medium theory, the layer permittivity can then be chosen to control the location of the bandpass regions.

Finally, the solutions have been generalized to real materials with complex refractive indices and successfully predict all of the observed resonances, even when the waves inside the metal are no longer purely evanescent.

\section{ACKNOWLEDGMENTS}

The authors acknowledge the Engineering and Physical Sciences Research Council (EPSRC) and Sharp Laboratories Europe for their financial support.

\section{REFERENCES}

1. T. W. Ebbesen, H. J. Lezec, H. F. Ghaemi, T. Thio, and P. A Wolff, "Extraordinary optical transmission through subwavelength hole arrays," Nature 391, 667-669 (1998).

2. H. F. Ghaemi, T. Thio, D. E. Grupp, T. W. Ebbesen, and H. J. Lezec, "Surface plasmons enhance optical transmission through subwavelength holes," Phys. Rev. B 58, 6779-6782 (1998).

3. M. Scalora, M. J. Bloemer, and C. M. Bowden, "Laminated photonic band structures with high conductivity and high transparency: metals under a new light," Opt. Photonics News 10, 23-27 (1998).

4. M. Scalora, M. J. Bloemer, A. S. Pethel, J. P. Dowling, C. M. Bowden, and A. S. Manka, "Transparent, metallo-dielectric, one-dimensional, photonic band-gap structures," J. Appl. Phys. 83, 2377-2383 (1998).

5. J. M. Bendickson, J. P. Dowling, and M. Scalora, "Analytic expressions for the electromagnetic mode density in finite, one-dimensional, photonic band-gap structures," Phys. Rev. E 53, 4107-4121 (1996).

6. M. C. Larciprete, C. Sibilia, S. Paolini, and M. Bertolotti, "Accessing the optical limiting properties of metallodielectric photonic band gap structures," J. Appl. Phys. 93, 5013-5017 (2003).

7. M. Scalora, G. D’Aguanno, N. Mattiucci, M. J. Bloemer, D. de Ceglia, M. Centini, A. Mandatori, C. Sibilia, N. Akozbek, M. G. Cappeddu, M. Fowler, and J. W. Haus, "Negative refraction and sub-wavelength focusing in the visible range using transparent metallodielectric stacks," Opt. Express 15, 508-523 (2007).

8. J. B. Pendry, "Negative refraction makes a perfect lens," Phys. Rev. Lett. 85, 3966-3969 (2000).

9. N. Fang, H. Lee, C. Sun, and C. X. Zhang, "Sub-diffractionlimited optical imaging with a silver superlens," Science 308, 534-537 (2005).

10. K. J. Webb and M. Yang, "Subwavelength imaging with a multilayer silver film structure," Opt. Lett. 31, 2130-2132 (2006).

11. C. Sibilia, I. S. Nefedov, M. Scalora, and M. Bertolotti, 
"Electromagnetic mode density for finite quasi-periodic structures," J. Opt. Soc. Am. B 15, 1947-1952 (1998).

12. A. Bichri, J. Lafait, and H. Welsch, "Visible and infrared optical properties of $\mathrm{Ag} / \mathrm{SiO} 2$ multilayers: radiative virtual modes and coupling effects," J. Phys.: Condens. Matter 5, 7361-7374 (1993).

13. A. Bichri, J. Lafait, H. Welsch, and M. Abd-Lefdil,
"Characterization of Berreman modes in metal/dielectric and multilayers," J. Phys.: Condens. Matter 9, 6523-6532 (1997).

14. A. P. Hibbins, M. J. Lockyear, and J. R. Sambles, "The resonant electromagnetic fields of an array of metallic slits acting as Fabry-Perot cavities," J. Appl. Phys. 99, 124903 (2006). 